\title{
Dielectric Properties and the Phase Transition of Pure and Cerium Doped Calcium-Barium-Niobate
}

\author{
Alexander Niemer ${ }^{1}$, Rainer Pankrath ${ }^{1}$, Klaus Betzler ${ }^{1}$, Manfred Burianek ${ }^{2}$, Manfred Muehlberg ${ }^{2}$ \\ ${ }^{1}$ Department of Physics, University of Osnabrück, Osnabrück, Germany; ${ }^{2}$ Institute of Crystallography, University of Cologne, Co- \\ logne, Germany. \\ Email: Klaus.Betzler@uos.de
}

Received December $2^{\text {nd }}, 2011$; revised February $25^{\text {th }}, 2012$; accepted March $2^{\text {nd }}, 2012$

\begin{abstract}
The complex dielectric constant of pure and cerium doped calcium-barium-niobate (CBN) was studied at frequencies 20 $\mathrm{Hz} \leq f \leq 1 \mathrm{MHz}$ in the temperature range $300 \mathrm{~K} \leq T \leq 650 \mathrm{~K}$ and compared with the results for the well known ferroelectric relaxor strontium-barium-niobate (SBN). By the analysis of the systematically taken temperature and frequency dependent measurements of the dielectric constant the phase transition characteristic of the investigated materials was evaluated. From the results it must be assumed that CBN shows a slightly diffuse phase transition without relaxor behavior. Doping with cerium yields a definitely different phase transition characteristic with some indications for a relaxor type ferroelectric material, which are common from SBN.
\end{abstract}

Keywords: 77.80.-e Ferroelectricity and Antiferroelectricity; 77.80.Jk Relaxor Ferroelectrics; 77.84.Ek Niobates and Tantalates; 77.22.Ch Permittivity (Dielectric Function)

\section{Introduction}

The ferroelectric material strontium-barium-niobate $\left(\mathrm{Sr}_{\mathrm{x}} \mathrm{Ba}_{1-\mathrm{x}} \mathrm{Nb}_{2} \mathrm{O}_{6}, \mathrm{SBN}\right)$ is well known for its excellent pyroelectric, piezoelectric, electrooptic, acoustooptic, photorefractive and non-linear optical properties [1-4]. But SBN exhibits a low phase transition temperature of about $353 \mathrm{~K}$ for the congruently melting composition with 61 mole\% strontium content (SBN:61) which hampers its applicability.

By the search for an alternative for SBN the isostructural material calcium-barium-niobate $\left(\mathrm{Ca}_{\mathrm{x}} \mathrm{Ba}_{1-\mathrm{x}} \mathrm{Nb}_{2} \mathrm{O}_{6}\right.$, $\mathrm{CBN}$ ) was found. $\mathrm{CBN}$ is a novel tungsten bronze which was first grown as single crystals a few years ago [5]. CBN offers similar physical properties like SBN, so it is also well suited for a wide range of applications. One difference is that CBN has an about $200 \mathrm{~K}$ higher phase transition temperature for the congruently melting composition with $28 \mathrm{~mole} \%$ of calcium (CBN:28) [5]. This is one of the main advantages of the material compared to SBN. The existence region of CBN with $0.2 \leq \mathrm{x} \leq 0.4$ of calcium content is smaller than the region for SBN with $0.32 \leq \mathrm{x} \leq 0.82$ for experimentally realized compositions $[6,7]$.

In literature it was found that CBN does not show a clear relaxor like behavior as SBN [8]. To investigate the phase transition behavior in more detail, in this work the dielectric properties of pure and cerium doped CBN are studied. The measurements of the dielectric constant and a comparison to the results of SBN are used to study the character of the phase transition of CBN. For SBN it is well known, that the material shows a relaxor type phase transition and that there is an influence of the concentration and type of rare earth dopants on the phase transition temperature and characteristic [9-12]. Previous investigations on CBN were mainly done on undoped crystals without methodical investigation of the dielectric constant. This paper presents the systematical measurement and analysis of the temperature and frequency dependency of the dielectric constant of CBN, cerium doped $\mathrm{CBN}(\mathrm{CBN}$ :Ce) and a comparison to SBN to get a characterization of the phase transition of $\mathrm{CBN}$ for the doped case, too.

\section{Experimental Details}

All investigated crystals, pure CBN, cerium doped CBN, and SBN were grown by the Czochralski technique from the congruently melting composition with $\mathrm{x}=0.28$ and $\mathrm{x}$ $=0.61$, respectively. The cerium doped samples were grown from a melt with the congruently melting composition and a cerium content of $1 \%$. For the measurements c-cut samples were used. The thickness of the samples was in the range of 1 to $4 \mathrm{~mm}$ and the c-faces were polished to optical quality. To electrically contact the samples the c-faces were covered with silver paste and then 
the samples with the silver paste electrodes were heated up to $670 \mathrm{~K}$ to get reproducible results. Otherwise one would measure side effects from the electrodes. The complex dielectric constant $\varepsilon=\varepsilon^{\prime}+i \varepsilon^{\prime \prime}$ was calculated from the values of the capacity and dissipation factor measured with an Hewlett-Packard 4284A LCR-meter in the temperature range from 300 to $650 \mathrm{~K}$ for the CBN samples and from 300 to $460 \mathrm{~K}$ for SBN. The measurements were performed in a frequency range from $20 \mathrm{~Hz}$ to $1 \mathrm{MHz}$ and taken under isothermal conditions. To ensure that, the CBN samples were heated with a low rate of $20 \mathrm{~K} / \mathrm{h}$ with respect to their larger thickness of 3 $4 \mathrm{~mm}$. The SBN samples were only about $1 \mathrm{~mm}$ thick and were therefore heated with a higher rate of $60 \mathrm{~K} / \mathrm{h}$ by a Peltier element. To achieve the higher temperatures, a high precision laboratory oven was used. As electrical field for the measurements about $3 \mathrm{~V} / \mathrm{mm}$ were used to minimize the influence on the sample and to obtain minimal noise.

\section{Results and Discussion}

The experimental results for the complex dielectric constant of CBN, CBN:Ce and SBN are shown in Figures 1-3. Both materials $\mathrm{CBN}$ and $\mathrm{CBN}$ :Ce show a ferroelectric phase transition indicated by the peak in the real part of the dielectric constant, as it is known for SBN.

The phase transition temperature was estimated as the peak temperature at a frequency of $20 \mathrm{~Hz}$. For CBN and CBN:Ce phase transition temperatures of $552 \mathrm{~K}$ and $462 \mathrm{~K}$, respectively, were measured, which are considerably higher than the value of $354 \mathrm{~K}$ for SBN. The cerium doping causes a lowering of the phase transition temperature of about $76 \mathrm{~K}$ compared to undoped CBN. Such a lowering of the phase transition temperature by doping with rare earth elements is known from $\operatorname{SBN}[12,13]$.

Regarding the absolute value of $\varepsilon^{\prime}$ it has to be pointed out that the values for $\mathrm{CBN}$ are about forty times larger than the values of SBN and the values of $\mathrm{CBN}$ :Ce are still by a factor of four greater. The absolute values for $\varepsilon^{\prime \prime}$ show nearly the same behavior.

The frequency dependency of $\varepsilon^{\prime}$ and $\varepsilon^{\prime \prime}$ is shown as an example for the case of CBN:Ce in the inset of Figure 2. From low to high frequencies the value of $\varepsilon_{m}^{\prime}$ decreases and starts to increase at very high frequencies again. $\varepsilon_{m}^{\prime \prime}$ shows qualitatively the same behavior but there is a more dramatic increase in the high frequency range.

All samples show a relatively broad peak in the dielectric constant around the phase transition temperature (Figures 1-3) which is typical for relaxor ferroelectrics or ferroelectrics with a diffuse phase transition $[3,14]$. But there are also significant differences in the behavior of CBN compared to the reference material SBN. For a better comparison Figure 4 shows the normalized dielectric

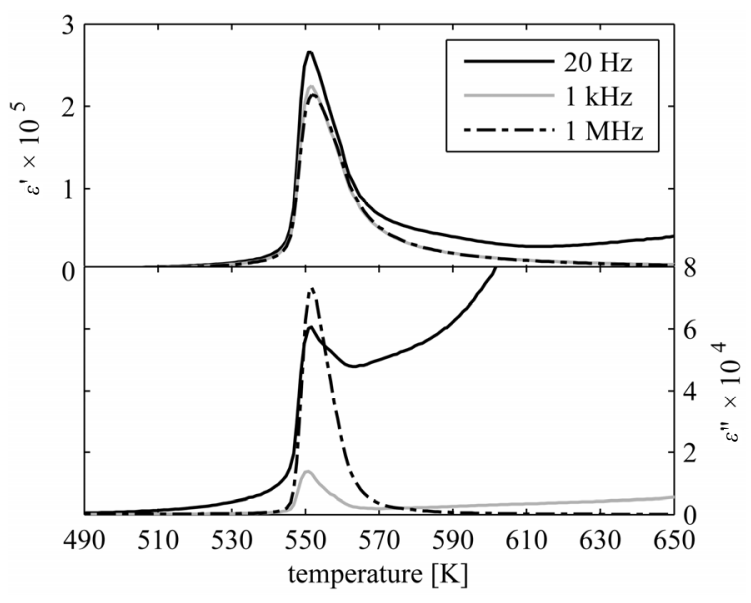

Figure 1. Temperature dependency of the real $\left(\varepsilon^{\prime}\right)$ and imaginary $\left(\varepsilon^{\prime \prime}\right)$ part of the dielectric constant for CBN:28.

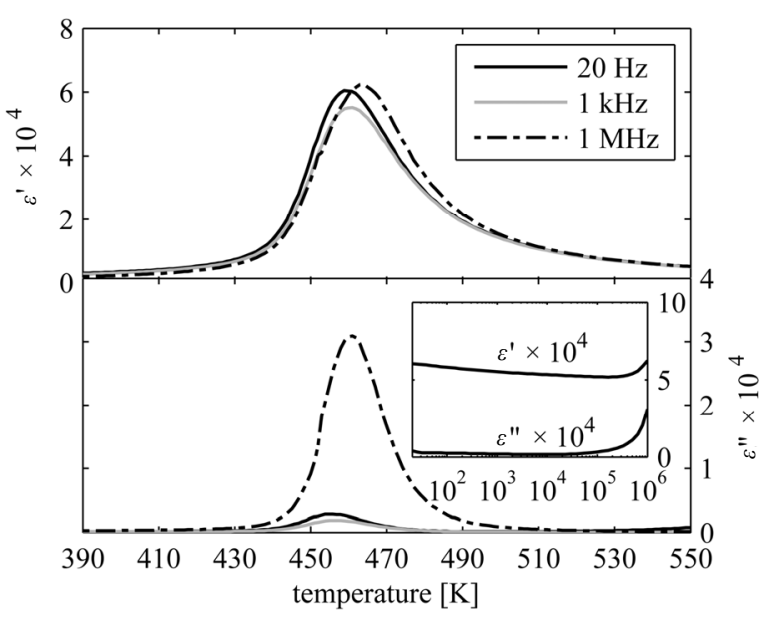

Figure 2. Temperature dependency of the real $\left(\varepsilon^{\prime}\right)$ and imaginary $\left(\varepsilon^{\prime \prime}\right)$ part of the dielectric constant for CBN:Ce. The inset shows the values of $\varepsilon^{\prime}$ and $\varepsilon^{\prime \prime}$ as a function of frequency.

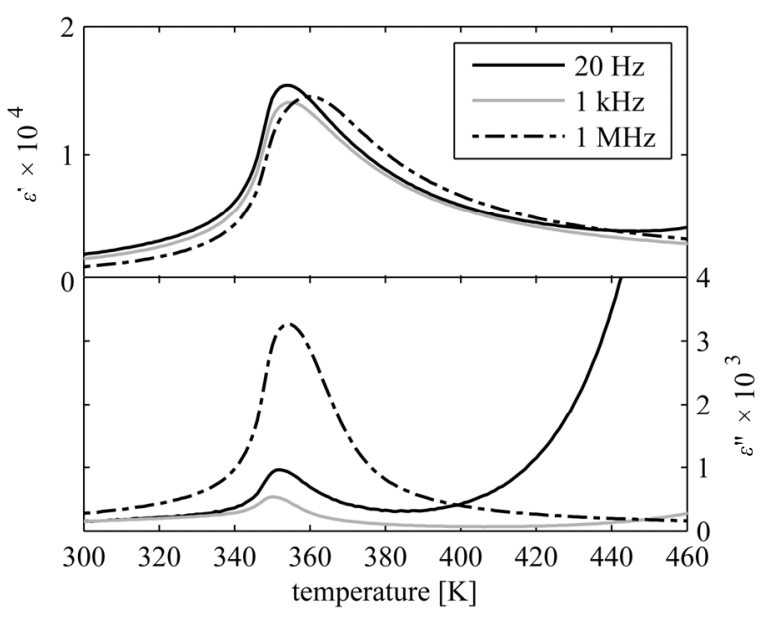

Figure 3. Temperature dependency of the real $\left(\varepsilon^{\prime}\right)$ and imaginary $\left(\varepsilon^{\prime \prime}\right)$ part of the dielectric constant for SBN:61. 
constant $\varepsilon^{\prime} / \varepsilon_{m}^{\prime}$ for all investigated samples. The peak in the real part of the dielectric constant of undoped CBN exhibits a smaller value of the full width at half maximum (FWHM) than the one for SBN. By comparison with the cerium doped sample it is evidenced that the doping induces a broadening of the peak in the dielectric constant which indicates a more diffuse phase transition characteristic of the material $[10,15]$. It can be concluded that the dopant has a strong influence on the characteristic of the ferroelectric phase transition of CBN.

The FWHM of the normalized dielectric constant can be used to determine the degree of diffuseness of the phase transition, higher values of FWHM mean a higher degree of diffuseness [15]. The frequency dependence of the FWHM values is shown in Figure 5. The values of the FWHM for pure CBN are about four times smaller than for SBN and indicate a phase transition with a very low degree of diffuseness, whereas for SBN a high degree of diffuseness of the phase transition is known. CBN:Ce shows a diffuse phase transition where the values of the FWHM are only about 1.5 times smaller than the ones for SBN. For CBN and CBN:Ce no frequency dependency of the FWHM can be observed, for SBN, however, an increase of the FWHM with the frequency is found.

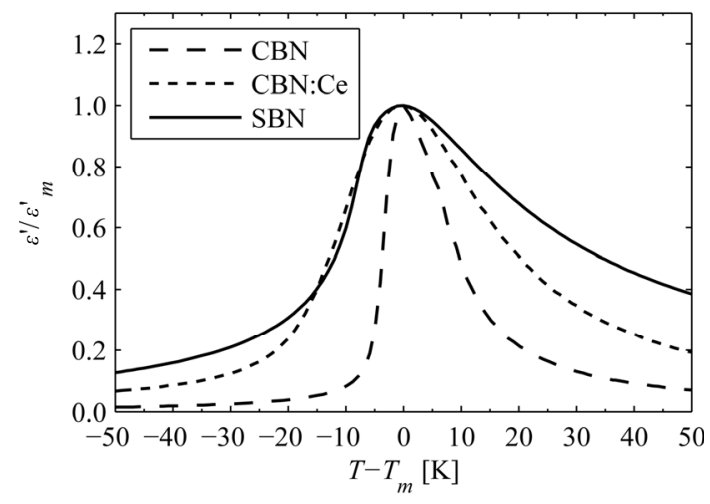

Figure 4. The normalized dieelectric constant $\varepsilon^{\prime} / \varepsilon_{m}^{\prime}$ around the peak-temperature $T_{m}$ at a frequency of $5 \mathrm{kHz}$.

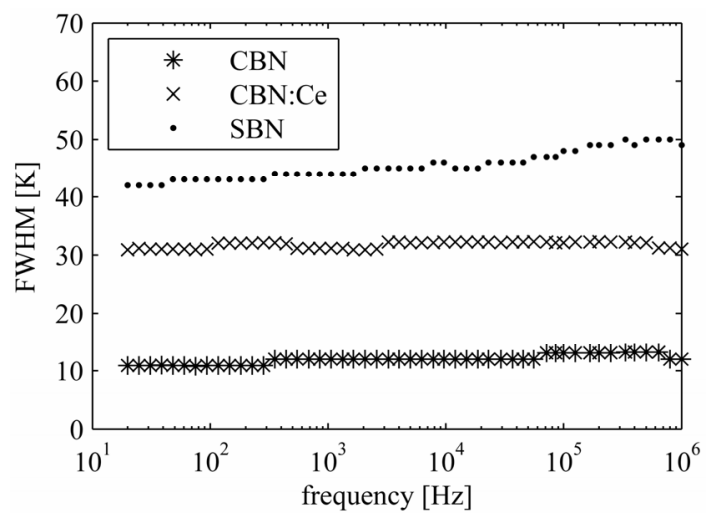

Figure 5. Frequency dependent plot of the FWHM for the investigated samples.
In ferroelectric relaxors a dependency of the peak temperature $T_{m}$ on the measurement frequency is expected [16], which can be described by the Vogel-Fulcher law [17-19]:

$$
f=f_{0} \exp \left(\frac{T_{0}}{T_{m}-T_{f}}\right)
$$

The best fitting parameters are listed in Table $\mathbf{1}$ and the measurement data and results of the fit are presented in Figure 6. For CBN:Ce and SBN the Vogel-Fulcher law fits excellent to the experimental results. For CBN a fit of the Vogel-Fulcher equation is not reasonable because there is no frequency dependence of $T_{m}$ in the investigated region from $20 \mathrm{~Hz}$ to $1 \mathrm{MHz}$. This evidences that $\mathrm{CBN}$ shows no or only very weak relaxor behavior, but nevertheless a diffuse phase transition because of the broadening of the peak in the dielectric constant shown above. For comparison, also the phase transition temperatures $T_{c}$ are listed in Table 1 -defined as the peak temperatures $T_{m}$ of $\varepsilon^{\prime}$ measured at lowest frequencies. For both samples CBN:Ce and SBN, extrapolated freezing temperature $T_{f}$ is lower than $T_{c}$ which indicates that the region of glassy freezing is never reached but replaced by a quasi critical behavior. Such a behavior is known from literature for rare earth doped SBN and is considered to be typical for an Ising-type relaxor [10].

Table 1. Best fit parameters of the $T_{m}$ vs. $f$ data shown in Figure 6 to the Vogel-Fulcher law. For comparison, also the values of $T_{c}$ are shown.

\begin{tabular}{ccccc}
\hline sample & $f_{0}[\mathrm{~Hz}]$ & $T_{0}[\mathrm{~K}]$ & $T_{f}[\mathrm{~K}]$ & $T_{m}[\mathrm{~K}]$ \\
\hline $\mathrm{CBN}$ & - & - & - & 552 \\
$\mathrm{CBN}: \mathrm{Ce}$ & $4.59 \times 10^{6}$ & 5.9 & 459.5 & 462 \\
$\mathrm{SBN}$ & $1.22 \times 10^{8}$ & 40.5 & 351.3 & 354 \\
\hline
\end{tabular}

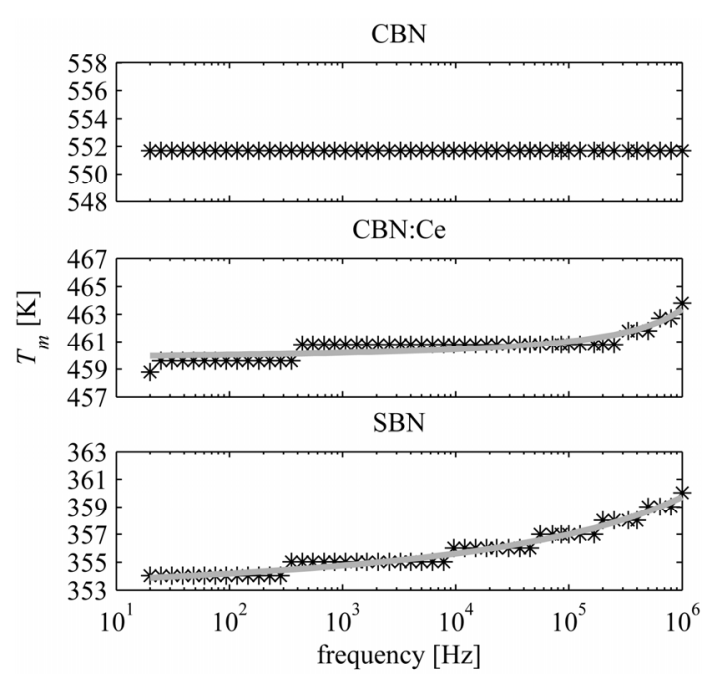

Figure 6. Frequency dependency of the peak-temperature of $\varepsilon^{\prime}\left(T_{m}\right)$ and fit according to Vogel-Fulcher's law described by the solid lines. 
The parameter $T_{0}=E_{a} / k_{B}$ where $k_{B}$ is Boltzmann's constant, describes the average activation energy. The activation energy for $\mathrm{CBN}$ :Ce is nearly ten times smaller than the one for SBN. Higher values of this energy are an indication for a rougher landscape of free energy of the system. Doping with cerium in SBN leads to higher values of $T_{0}[20]$ thus it can be concluded that CBN:Ce has a mainly flat landscape of free energy.

The values of the calculated frequency $f_{0}$ are far below the soft mode frequency, which is in the order of $10^{10}$ $10^{11} \mathrm{~Hz}$. Following the argumentation in [20] random field correlated polar clusters are assumed to be responsible for this untypical behavior.

For ferroelectric materials with a first or second order phase transition described by Landau's theory the behavior of the reciprocal dielectric constant above the CurieTemperature $\left(T_{c}\right)$ can be described by the Curie-Weiss Law. But for ferroelectrics with a diffuse or relaxor like phase transition the normal Curie-Weiss Law can only be used in an adequate distance from $T_{m}$ [21]. Near $T_{m}$ the reciprocal dielectric constant can be better described by a modified Curie-Weiss Law [22,23]:

$$
\frac{1}{\varepsilon^{\prime}}-\frac{1}{\varepsilon_{m}^{\prime}}=\frac{\left(T-T_{m}\right)^{\gamma}}{C_{C}}
$$

This modified version transforms to the normal $\mathrm{Cu}$ rie-Weiss Law for conventional ferroelectrics because for this materials one gets $\gamma=1$; for materials with a complete diffuse phase transition $\gamma$ has values near 2. The best fitting of Equation (2) to the experimental results is shown in Figure 7. From the calculated values of $\gamma$ it can be seen that all investigated materials show a partially diffuse phase transition because $\gamma$ has a value around 1.5. The value of $\gamma$ for $\mathrm{CBN}$ is slightly smaller than for $\mathrm{CBN}$ :Ce which is nearly the same evaluated for SBN, but there is not such a clear difference like in the results for

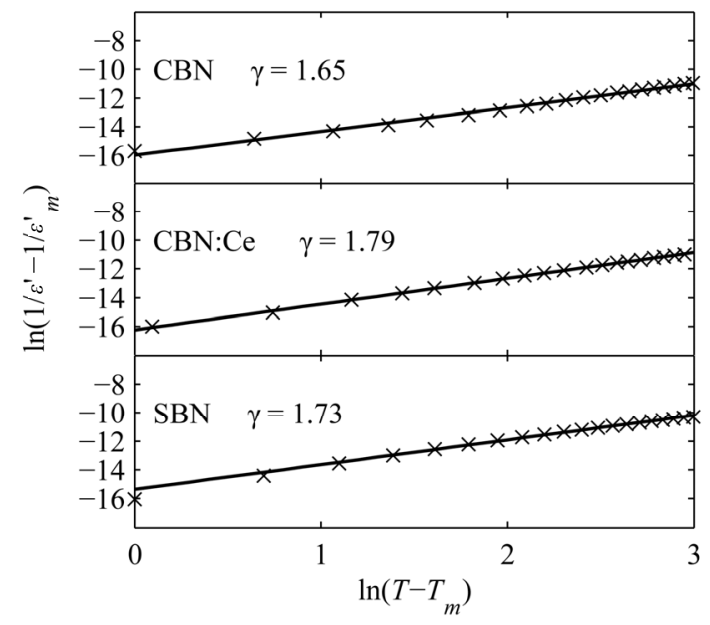

Figure 7. Frequency dependent plot of the FWHM for the investigated samples. the FWHM. Thence the FWHM should not be used as the sole property for the characterization of the phase transition.

\section{Conclusions}

The dielectric constant data of CBN, CBN:Ce, SBN show, that the phase transition characteristic of the three materials is considerably different. The analysis of the fullwidth at half maximum yields a slightly diffuse phase transition characteristic for $\mathrm{CBN}$ and a distinct diffuseness of the phase transition for CBN:Ce which is in the range of the phase transition of SBN. From the results of the Vogel-Fulcher law can be obtained that CBN shows no relaxor behavior whereas CBN:Ce shows like SBN a clear frequency dependency of the peak temperature which follows Vogel-Fulcher's law. Such a deviation is a typical sign of ferroelectric relaxors [10]. Further it was shown, that $\mathrm{CBN}$ :Ce has a very flat free energy landscape compared to the well-known ferroelectric relaxor SBN. By the reciprocal dielectric constant and the critical exponent calculated from it was shown that all investigated materials show a partially diffuse phase transition, as the critical exponent of $\mathrm{CBN}$ is marginally smaller than the one for $\mathrm{CBN}$ :Ce or SBN.

It can be concluded that CBN shows a slightly diffuse phase transition without the typical relaxor behavior whereas CBN:Ce shows a distinctly diffuse phase transition with a typical behavior for ferroelectric relaxors like SBN. Thus, as in the case of SBN, doping with rare earth elements has a strong influence on the phase transition characteristic of CBN.

\section{Acknowledgements}

Financial support from the Deutsche Forschungsgemeinschaft and the federal state of Niedersachsen within the graduate college GRK 695 "Nonlinearities of Optical Materials" is gratefully acknowledged.

\section{REFERENCES}

[1] A. S. Kewitsch, M. Segev, A. Yariv, G. J. Salamo, T. W. Towe, E. J. Sharp and R. R. Neurgaonkar, "Tunable Quasi-Phase Matching Using Dynamic Ferroelectric Domain Gratings Induced by Photorefractive Space - Charge Fields," Applied Physics Letters, Vol. 64, No. 23, 1994, pp. 3068-3070. doi:10.1063/1.111349

[2] R. R. Neurgaonkar, W. F. Halla, J. R. Olivera, W. W. Hoa and W. K. Corya, "Tungsten Bronze $\mathrm{Sr}_{1-\mathrm{x}} \mathrm{Ba}_{\mathrm{x}} \mathrm{Nb}_{2} \mathrm{O}_{6}$ : A Case History of Versatility," Ferroelectrics, Vol. 87, No. 1, 1988, pp. 167-179. doi:10.1080/00150198808201379

[3] L. E. Cross, "Relaxor Ferroelectrics," Ferroelectrics, Vol. 76, No. 1, 1987, pp. 241-267. doi: $10.1080 / 00150198708016945$

[4] M. Horowitz, A. Bekker and B. Fischer, "Broadband 
Second-Harmonic Generation in $\mathrm{Sr}_{\mathrm{x}} \mathrm{Ba}_{1-\mathrm{x}} \mathrm{Nb}_{2} \mathrm{O}_{6}$ by Spread Spectrum Phase Matching with Controllable Domain Gratings," Applied Physics Letters, Vol. 62, No. 21, 1993, pp. 2619-2621. doi:10.1063/1.109264

[5] M. Eßer, M. Burianek, D. Klimm and M. Mühlberg, "Single Crystal Growth of the Tetragonal Tungsten Bronze $\mathrm{Ca}_{\mathrm{x}} \mathrm{Ba}_{1-\mathrm{x}} \mathrm{Nb}_{2} \mathrm{O}_{6}(\mathrm{x}=0.28$; $\mathrm{CBN}-28)$," Journal of Crystal Growth, Vol. 240, No. 1-2, 2002, pp.1-5. doi:10.1016/S0022-0248(02)00868-0

[6] M. Eßer, M. Burianek, P. Held, J. Stade, S. Bulut, C. Wickleder and M. Mühlberg, "Optical Characterization and Crystal Structure of the Novel Bronzetype $\mathrm{Ca}_{\mathrm{x}} \mathrm{Ba}_{1-\mathrm{x}} \mathrm{Nb}_{2} \mathrm{O}_{6}$ $(\mathrm{x}=0.28 ; \mathrm{CBN}-28)$," Crystal Research and Technology, Vol. 38, No. 6, 2003, pp. 457-464. doi:10.1002/crat.200310057

[7] M. Ulex, R. Pankrath and K. Betzler, "Growth of Strontium Barium Niobate: The LIquidus-Solidus Phase Diagram," Journal of Crystal Growth, Vol. 271, No. 1-2, 2004, pp. 128-133. doi:10.1016/j.jcrysgro.2004.07.039

[8] Y. J. Qi, C. J. Lu, J. Zhu, X. B. Chen, H. L. Song, H. J. Zhang and $\mathrm{X}$. G. Xu, "Ferroelectric and Dielectric Properties of $\mathrm{Ca}_{\mathrm{x}} \mathrm{Ba}_{1-\mathrm{x}} \mathrm{Nb}_{2} \mathrm{O}_{6}$ Single Crystals of Tungsten Bronzes Structure," Applied Physics Letters, Vol. 87, No. 8, 2005, Article ID 082904. doi:10.1063/1.2010614

[9] O. Kersten, A. Rost and G. Schmidt, "Dielectric Dispersion of Relaxor Ferroelectrics (SBN 75 and PLZT 8/65/35)," Physica Status Solidi A, Vol. 75, No. 2, 1983, pp. 495500. doi:10.1002/pssa.2210750220

[10] I. A. Santos, D. U. Spinola, D. Garcia and J. A. Eiras, "Dielectric Behavior and Diffuse Phase Transition Features of Rare Earth Doped $\mathrm{Sr}_{0.61} \mathrm{Ba}_{0.39} \mathrm{Nb}_{2} \mathrm{O}_{6}$ Ferroelectric Ceramic," Journal of Applied Physics, Vol. 92, No. 6, 2002, pp. 3251-3256. doi:10.1063/1.1481210

[11] S. Kuroda and K. Kubota, "Diffuse Phase Transition in Rare Earth Ion Doped SBN," Journal of Physics and Chemistry of Solids, Vol. 42, No. 7, 1981, pp. 573-577. doi:10.1016/0022-3697(81)90104-9

[12] T. Volk, L. Ivleva, P. Lykov, N. Polozkov, V. Salobutin, R. Pankrath and M. Wöhlecke, "Effects of Rare-Earth Impurity Doping on the Ferroelectric and Photorefractive Properties of Strontium-Barium Niobate Crystals," Optical Materials, Vol. 18, No. 1, 2001, pp. 179-182. doi:10.1016/S0925-3467(01)00162-8

[13] I.-I. Oprea, U. Voelker, A. Niemer, R. Pankrath, S. Pod- lozhenov and K. Betzler, "Influence of Erbium Doping on Phase Transition and Optical Properties of Strontium Barium Niobate," Optical Materials, Vol. 32, No. 1, 2009, pp. 30-34. doi:10.1016/j.optmat.2009.05.015

[14] L. E. Cross, "Relaxor Ferroelectrics: An Overview," Ferroelectrics, Vol. 151, No. 1, 1994, pp. 305-320. doi:10.1080/00150199408244755

[15] J. Kim, M. Jang, I. Kim and K. Lee, "Niobium Doping Effects and Ferroelectric Relaxor Behavior of Bismuth Lantanium Titanate," Journal of Electroceramics, Vol. 17, No. 2-4, 2006, pp. 129-133. doi:10.1007/s10832-006-5410-9

[16] A. K. Tagantsev, "Vogel-Fulcher Relationship for the Dielectric Permittivity of Relaxor Ferroelectrics," Physical Review Letters, Vol. 72, No. 7, 1994, pp. 1100-1103. doi:10.1103/PhysRevLett.72.1100

[17] G. Fulcher, "Analysis of Recent Measurements of the Viscosity of Glasses," Journal of the American Ceramic Society, Vol. 8, No. 6, 1925, pp. 339-355. doi:10.1111/j.1151-2916.1925.tb16731.x

[18] F. Chu, I. M. Reaney and N. Setter, "Spontaneous (ZeroField) Relaxor-to-Ferroelectric-Phase Transition in Disordered $\mathrm{Pb}\left(\mathrm{Sc}_{1 / 2} \mathrm{Nb}_{1 / 2}\right) \mathrm{O}_{3}$," Journal of Applied Physics, Vol. 77, No. 4, 1995, pp. 1671-1676. doi:10.1063/1.358856

[19] D. H. Vogel, "Das Temperaturabhaengigkeitsgesetz der Viskositaet von Fluessigkeiten," Physikalische Zeitschrift, Vol. 22, 1921, p. 645.

[20] J. Dec, W. Kleemann, T. Woike and R. Pankrath, "Phase Transitions in $\mathrm{Sr}_{0.61} \mathrm{Ba}_{0.39} \mathrm{Nb}_{2} \mathrm{O}_{6}: \mathrm{Ce}^{3+}$ : I. Susceptibility of Clusters and Domains," European Physical Journal B, Vol. 14, No. 4, 2000, pp. 627-632. doi:10.1007/s100510051071

[21] W. H. Huang, D. Viehland and R. R. Neurgaonkar, "Anisotropic Glasslike Characteristics of Strontium Barium Niobat Relaxors," Journal of Applied Physics, Vol. 76, No. 1, 1994, pp. 490-496. doi:10.1063/1.357100

[22] K. Uchino and S. Nomura, "Critical Exponents of the Dielectric Constants in Diffused-Phase-Transition Crystals," Ferroelectrics Letters, Vol. 44, 1982, pp. 55-61. doi: 10.1080/07315178208201875

[23] Y. Guo, K. Kakimoto and H. Ohsato, "Dielectric and Piezoelectric Properties of Lead-Free $\left(\mathrm{Na}_{0.5} \mathrm{~K}_{0.5}\right) \mathrm{NbO}_{3^{-}}$ $\mathrm{SrTiO}_{3}$ Ceramics," Solid State Communications, Vol. 129, No. 5, 2004, pp. 279-284. doi:10.1016/j.ssc.2003.10.026 